\title{
EXTENSIONS OF STRICT POLYNOMIAL FUNCTORS
}

\author{
BY MARCIN CHAŁUPNIK ${ }^{1}$
}

\begin{abstract}
We compute Ext-groups between Frobenius twists of strict polynomial functors. The main result concerns the groups $\operatorname{Ext}_{\mathcal{P}}^{*}\left(D^{d(i)}, F^{(i)}\right)$ where $D^{d}$ is the divided power functor, and $F$ is an arbitrary functor. These groups are shown to be isomorphic to $F\left(A_{i}\right)$ for certain explicitly described graded space $A_{i}$. We also calculate the groups $\operatorname{Ext}_{\mathcal{P}}^{*}\left(W_{\mu}^{(i)}, S_{\lambda}^{(i)}\right)$ where $W_{\mu}$ and $S_{\lambda}$ are respectively the Weyl and Schur functors associated to diagrams $\mu, \lambda$ of the same weight.
\end{abstract}

(C) 2005 Elsevier SAS

RÉSUMÉ. - Nous considérons les modules sur l'algèbre de Schur sur un corps fini sous la forme de foncteurs polynomiaux, et calculons leurs groupes d'extensions, qui sont aussi la valeur générique de la cohomologie des groupes linéaires. Les résultats principaux concernent les groupes $\operatorname{Ext}_{\mathcal{P}}^{*}\left(D^{d(i)}, F^{(i)}\right)$, extensions entre le twist de Frobenius d'un foncteur $F$ quelconque et le twist d'une puissance divisée, et les groupes $\operatorname{Ext}_{\mathcal{P}}^{*}\left(W_{\mu}^{(i)}, S_{\lambda}^{(i)}\right)$, extensions entre le twist d'un foncteur de Schur et d'un foncteur de Weyl.

(C) 2005 Elsevier SAS

\section{Introduction}

Computing Ext-groups between discrete or rational $G L_{n}$-modules over a field $\mathbf{k}$ of positive characteristic had been known to be a very difficult problem for a long time. Only recently, introducing a suitable category of functors $\mathcal{F}$ in [14] has changed the situation significantly. As it was demonstrated in [11-13], it is possible to make effective computations of Ext-groups in the category $\mathcal{F}$ (and even more effective in its algebrogeometric modification $\mathcal{P}$ ). In a meantime, in a series of papers $[2,16-18,13,11]$ there was established a close connection between the functor categories $\mathcal{F}, \mathcal{P}$ and various module categories. More specifically, it was shown in [13, Corollary 3.13] that for any strict polynomial functors $F, G$ of degree $d$ and $n \geqslant d$

$$
\operatorname{Ext}_{\mathcal{P}}^{*}(F, G) \simeq \operatorname{Ext}_{G L_{n} \text {-mod }}^{*}\left(F\left(\mathbf{k}^{n}\right), G\left(\mathbf{k}^{n}\right)\right),
$$

where the Ext-groups at the right-hand side are taken in the category of rational representations of an algebraic group $G L_{n}$. In the present paper we focus our attention on quite specific strict polynomial functors: on Frobenius twists (cf. [13, Section 1]). The reason is that in this case the Ext-groups in $\mathcal{P}$ are also related to the Ext-groups between discrete modules. Namely, a result of Betley [2] and Suslin [11, Appendix] implies that for any strict polynomial functors $F, G$ of degree $d$ over a field $\mathbf{k}$ with at least $d$ elements, and for any integer $k$,

$$
\operatorname{Ext}_{\mathcal{P}}^{k}\left(F^{(i)}, G^{(i)}\right) \simeq \operatorname{Ext}_{G L_{n}(\mathbf{k})-\bmod }^{k}\left(F\left(\mathbf{k}^{n}\right), G\left(\mathbf{k}^{n}\right)\right),
$$

\footnotetext{
${ }^{1}$ The author was partially supported by Białynicki-Birula Subsydium of Foundation of Polish Science and the polish scientific Grant (KBN) 1 P03A 00526.
} 
for large enough integers $i, n\left(F^{(i)}\right.$ stands here for the $i$-th Frobenius twist of a functor $F$ ). Thus we see that the Ext-groups in the category of strict polynomial functors are closely related to Ext-groups in some important categories. But the main point is that the calculations in $\mathcal{P}$ are drastically easier than those in module categories. Such calculations were started in [13] where (adapting ideas of [12] to the context of the category $\mathcal{P}$ ) the groups $\operatorname{Ext}_{\mathcal{P}}^{*}\left(I^{(i)}, I^{(i)}\right)$ were computed. Already this result has a valuable application to $G L_{n}(\mathbf{k})$-modules, for after some additional work [13, Section 7] it leads to a computation of $H^{*}\left(G L_{n}\left(\mathbf{F}_{p}\right), M_{n}\left(\mathbf{F}_{p}\right)\right)$ for large $n$ (with action of $G L_{n}\left(\mathbf{F}_{p}\right)$ on matrices by conjugation), which is equivalent to the difficult results of Bökstedt [4] and Breen [5]. These computations were extended in [10,11,21].

The aim of the present paper is to generalize and systematize computations of Ext-groups in the functor category $\mathcal{P}$ by using methods of representation theory. We obtain a complete description of the Ext-groups for a large class of functors which strongly generalizes and puts into a uniform context known computations.

A direct inspiration for this work was the computation of $\operatorname{Ext}_{\mathcal{P}}^{*}\left(D^{d(i)}, S^{d(i)}\right)$ obtained in [11]. Since the tensor products of divided powers form a family of projective generators of $\mathcal{P}$ and the products of symmetric powers - of injective ones, one can hope for computations of Ext-groups for the Frobenius twists of functors of a more general form.

The main results are computations of $\operatorname{Ext}^{*}\left(D^{d(i)}, F^{(i)}\right)$ for an arbitrary $F$ (Theorem 4.3, Corollary 5.3) and $\operatorname{Ext}^{*}\left(F^{(i)}, G^{(i)}\right)$ for $F, G$ satisfying certain simple abstract condition (Theorem 4.4). The most important instance of Theorem 4.4 is for functors $F=W_{\mu}$ and $G=S_{\lambda}$ (respectively Weyl and Schur functors) for diagrams $\mu, \lambda$ of the same weight (Theorem 6.1). The language in which results of computations are given utilizes a concept of "symmetrization of functor" (see Section 3). This notion exploits a strong interplay between representations of the general linear group and the symmetric group coming from the action of these groups on the tensor power of a space, and may be thought of as generalization of a classic notion of symmetrization of representation.

This paper is a first part of my work on homological algebra in the category of functors. In the next article [7] I partially expand computations of Ext-groups between twisted Weyl and Schur functors to the case of diagrams of different weights. As it is not surprising for a reader of [11-13], an essential role in that work is played by the de Rham complex. Its appropriate generalization to the case of an arbitrary Young diagram has turned out to be an object complicated and interesting for its own. I investigate it in detail in a separate article [6]. The ideas of the present article are also a starting point of [8] where a complete description of Ext-groups between exponential functors is given.

\section{Recollections}

We start by collecting some basic facts concerning Young diagrams and functors one can associate to them.

\subsection{Diagrams}

A Young diagram $\lambda$ of weight $d$ is just a weakly decreasing sequence of positive integers $\left(\lambda_{1}, \ldots, \lambda_{l}\right)$ with $\sum_{j=1}^{l} \lambda_{j}=d=:|\lambda|$. We can associate to a Young diagram $\lambda$ the conjugate diagram $\tilde{\lambda}$ whose rows are columns of $\lambda$ (formally: $\widetilde{\lambda}_{k}=\#\left\{j: \lambda_{j} \leqslant k\right\}$ ). We shall consider the partial ordering of dominance on the set of Young diagrams. We say that $\lambda$ dominates $\mu$

$(\mu \unlhd \lambda)$ if for all $j$ we have $\sum_{i \leqslant j} \lambda_{i} \leqslant \sum_{i \leqslant j} \mu_{i}$. This partial ordering may be enriched to the total lexicographic ordering: $\mu \leqslant \lambda$ if for the least $i$ such that $\mu_{i} \neq \lambda_{i}$, we have $\mu_{i}>\lambda_{i}$. The

$4^{\mathrm{e}}$ SÉRIE - TOME $38-2005-\mathrm{N}^{\circ} 5$ 
direction of dominance and lexicographic relations looks strange, since the lesser diagram is the longer rows it has. The reason is that the terminology in our two main references: [1,9] is not consistent. I decided to follow the conventions of [1] when dealing with Schur functors etc., but I follow [9] with respect to the direction of orderings.

Given two diagrams $\mu \subseteq \lambda$ (i.e. $\mu_{j} \leqslant \lambda_{j}$ for all $j$ ), we may form a skew diagram $\lambda / \mu$ which should be imagined as a diagram $\lambda$ with deleted boxes belonging to $\mu$. Here is a picture for $(4,3,1) /(2,1)$

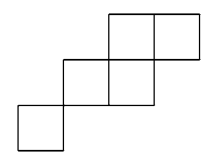

\subsection{Polynomial functors}

Throughout this paper $\mathbf{k}$ is a field of positive characteristic $p$ and $\mathcal{P}_{d}$ denotes the category of homogeneous strict polynomial functors of degree $d$ over $\mathbf{k}$ [13, Section 2]. All Extgroups are computed in $\mathcal{P}_{d}$ for appropriate $d$. We now recall certain important objects in $\mathcal{P}_{d}$. The most fundamental are: the $d$-th tensor power $I^{d}(V):=V^{\otimes d}$, the $d$-th symmetric power $S^{d}(V):=\left(V^{\otimes d}\right)_{\Sigma_{d}}$, the $d$-th divided power $D^{d}(V):=\left(V^{\otimes d}\right)^{\Sigma_{d}}$, (the last two functors are not isomorphic for $d \geqslant p$ ) and the $d$-th exterior power $\Lambda^{d}(V):=\left(V^{\otimes d}\right)^{\Sigma_{d}} \simeq\left(V^{\otimes d}\right)_{\Sigma_{d}}$ for the alternating action of $\Sigma_{d}$ on the tensor power (this definition needs a modification for $p=2$, we will discuss it in detail in the next section). There are also well-known transformations between these functors e.g. the inclusion $c_{d}: \Lambda^{d} \rightarrow I^{d}$ and the epimorphism $m_{d}: I^{d} \rightarrow S^{d}$.

Given a diagram $\lambda$ of weight $d$, we put $\Lambda^{\lambda}:=\Lambda^{\lambda_{1}} \otimes \cdots \otimes \Lambda^{\lambda_{l}}$ and $c_{\lambda}:=c_{\lambda_{1}} \otimes \cdots \otimes$ $c_{\lambda_{l}}: \Lambda^{\lambda} \rightarrow I^{d}$. In the same fashion we define $S^{\widetilde{\lambda}}$ and $m_{\widetilde{\lambda}}: I^{d} \rightarrow S^{\widetilde{\lambda}}$, but one should remember that $m_{\tilde{\lambda}}$ acts in a "conjugate manner" i.e. we gather the elements which have indices belonging to the same column (see [1, Section II.1]). We are now in a position to introduce a more complicated object. The Schur functor $S_{\lambda}$ is defined as the image of the composition $m_{\tilde{\lambda}} \circ c_{\lambda}$. It comes with two structural transformations: the epimorphism $\phi_{\lambda}: \Lambda^{\lambda} \rightarrow S_{\lambda}$ and monomorphism $\psi_{\lambda}: S_{\lambda} \rightarrow S^{\widetilde{\lambda}}$, which in extreme cases give isomorphisms $S_{(d)} \simeq \Lambda^{d}, S_{\left(1^{d}\right)} \simeq S^{d}$.

There is a useful contravariant duality in the category $\mathcal{P}_{d}$ called the Kuhn duality: $F^{\#}(V)=$ $\left(F\left(V^{*}\right)\right)^{*}$ where $V^{*}$ means the $\mathbf{k}$-dual space. It is easy to verify that $\left(D^{d}\right)^{\#} \simeq S^{d}$ while $\Lambda^{d}$ is selfdual. We will also consider the Kuhn duals of Schur functors which are called Weyl functors and denoted by $W_{\lambda}$. The independent definition of Weyl functor is, of course, as the image of the composition $D^{\widetilde{\lambda}} \rightarrow I^{d} \rightarrow \Lambda^{\lambda}$.

All these constructions may be applied to skew diagrams as well. Although skew Schur and Weyl functors $S_{\lambda / \mu}, W_{\lambda / \mu}$ play less important roles in the theory (in fact the LittlewoodRichardson rule [3] says that any skew Schur (respectively Weyl) functor has a filtration with a graded object being a sum of Schur (respectively Weyl) functors), they are often useful in inductive arguments.

The category $\mathcal{P}_{d}$ is endowed with certain abstract structure introduced in [9] called the structure of highest weight category (it follows from the fact that $\mathcal{P}_{d}$ is equivalent to the category of finitely generated modules over the Schur algebra $S(n, d)$ for any $n \geqslant d$ [13, Theorem 3.2], for which the structure of highest weight category was studied in detail e.g. in [19, Chapter 3]). We shall need two formal consequences of the fact that $\mathcal{P}_{d}$ is a highest weight category.

FACT 2.1. - For any skew diagrams $\mu / \mu^{\prime}, \lambda / \lambda^{\prime}, \operatorname{Ext}^{n}\left(W_{\lambda / \lambda^{\prime}}, S_{\mu / \mu^{\prime}}\right)=0$, for $n>0$. 
Proof. - It is shown at the beginning of the proof of [9, Theorem 3.11] that $\operatorname{Ext}^{1}\left(W_{\lambda}, S_{\mu}\right)=0$ (the Schur and Weyl functors $S_{\mu}, W_{\lambda}$ correspond respectively to $A(\mu)$ and $V(\lambda)$ in the abstract context considered in [9]) for all $\lambda, \mu$, but the authors point out that their argument also works for $\operatorname{Ext}^{n}$ for $n>1$. In order to extend our result to the case of skew diagrams it suffices to apply the Littlewood-Richardson rule [3] to both variables.

FACT 2.2. - If for some $n>0, \operatorname{Ext}^{n}\left(S_{\mu}, S_{\lambda}\right) \neq 0$, then $\mu \triangleright \lambda$.

Proof. - Again, this is shown in [9, Lemma 3.2(b)] only for $n=1$ but the proof carries over to all $n \geqslant 1$.

We finish this section by introducing the main technical tool, which will be used repeatedly in the next sections. This tool is the Decomposition Formula. Let $\mathcal{P}^{n}$ denote the category of strict polynomial functors in $n$ variables. The Decomposition Formula [1, Theorem II.4.11] provides an extremely useful filtration of a functor in two variables $S_{\lambda / \mu}(V \oplus W)$.

FACT 2.3 (Decomposition formula). - The bifunctor $S_{\lambda / \mu}(V \oplus W)$ has a filtration $M_{\alpha}(V, W)$ (for $\alpha$ satisfying $\mu \subseteq \alpha \subseteq \lambda$ ). The ordering in the filtration comes from the lexicographic ordering among $\alpha$. Its associated graded object is

$$
\bigoplus_{\mu \subseteq \alpha \subseteq \lambda} S_{\alpha / \mu}(V) \otimes S_{\lambda / \alpha}(W)
$$

Iterating this procedure we get a filtration of the $n$-functor $S_{\lambda / \mu}\left(V_{1} \oplus \cdots \oplus V_{n}\right)$.

COROLlaRY 2.4. - The functor in $n$ variables $S_{\lambda / \mu}\left(V_{1} \oplus \cdots \oplus V_{n}\right)$ has a filtration $M_{\mu \subseteq \alpha^{1} \subseteq \ldots \subseteq \alpha^{n-1} \subseteq \lambda}$, with ordering coming from the $n$-fold lexicographic ordering (i.e. to compare sequences $\left(\alpha^{1}, \ldots, \alpha^{n-1}\right) i\left(\alpha^{1}, \ldots, \alpha^{\prime n-1}\right)$ we pick the smallest $i$ such that $\alpha^{i} \neq \alpha^{\prime i}$ and compare lexicographically $\alpha^{i}$ and $\left.\alpha^{\prime i}\right)$. Its graded object is

$$
\bigoplus_{\mu \subseteq \alpha^{1} \subseteq \cdots \subseteq \alpha^{n-1} \subseteq \lambda} S_{\alpha^{1} / \mu}\left(V_{1}\right) \otimes \cdots \otimes S_{\alpha^{n-1} / \alpha^{n-2}}\left(V_{n-1}\right) \otimes S_{\lambda / \alpha^{n-1}}\left(V_{n}\right) .
$$

Of course, we get an analogous decomposition for twisted Schur functors and for Weyl functors. This filtration is a powerful tool in computations of Ext-groups, since as it was observed in [11, pp. 671-672], the evident adjoint functors between $\mathcal{P}$ and $\mathcal{P}^{n}$ yield an isomorphism

$$
\operatorname{Ext}_{\mathcal{P}}^{*}\left(F_{1} \otimes \cdots \otimes F_{n}, S_{\lambda / \mu}^{(i)}\right)=\operatorname{Ext}_{\mathcal{P}^{n}}^{*}\left(F_{1}\left(V_{1}\right) \otimes \cdots \otimes F_{n}\left(V_{n}\right), S_{\lambda / \mu}^{(i)}\left(V_{1} \oplus \cdots \oplus V_{n}\right)\right) ;
$$

I wrote down spaces $V_{1}, \ldots, V_{n}$ in the right-hand side of the formula to emphasize the dependence of the functor on $n$ variables. We recall from [11, p. 672], that the "Kunneth formula" gives an isomorphism

$$
\begin{aligned}
& \operatorname{Ext}_{\mathcal{P}^{n}}^{*}\left(F_{1}\left(V_{1}\right) \otimes \cdots \otimes F_{n}\left(V_{n}\right), S_{\alpha^{1} / \mu}^{(i)}\left(V_{1}\right) \otimes \cdots \otimes S_{\lambda / \alpha^{n-1}}^{(i)}\left(V_{n}\right)\right) \\
& \quad=\operatorname{Ext}_{\mathcal{P}}^{*}\left(F_{1}, S_{\alpha^{1} / \mu}^{(i)}\right) \otimes \cdots \otimes \operatorname{Ext}_{\mathcal{P}}^{*}\left(F_{n}, S_{\lambda / \alpha^{n-1}}^{(i)}\right) .
\end{aligned}
$$

Thus the Decomposition Formula leads to a spectral sequence, which we will call the Decomposition Spectral Sequence.

$4^{\text {e }}$ SÉRIE - TOME $38-2005-\mathrm{N}^{\circ} 5$ 
COROLLARY 2.5. - There exists a spectral sequence converging to $\operatorname{Ext}_{\mathcal{P}}^{*}\left(F_{1} \otimes \cdots \otimes F_{n}, S_{\lambda / \mu}^{(i)}\right)$, whose $E^{1}$-term has the form

$$
E_{i j}^{1}=\bigoplus_{i_{1}+\cdots+i_{n}=i+j} \operatorname{Ext}_{\mathcal{P}}^{i_{1}}\left(F_{1}, S_{\alpha^{1} / \mu}^{(i)}\right) \otimes \cdots \otimes \operatorname{Ext}_{\mathcal{P}}^{i_{n}}\left(F_{n}, S_{\lambda / \alpha^{n-1}}^{(i)}\right)
$$

where $j$ stands for a place of $\left(\alpha^{1}, \ldots, \alpha^{n-1}\right)$ in the $(n-1)$-fold lexicographic ordering. etc.

Analogous sequences also exist for $\operatorname{Ext}_{\mathcal{P}}^{*}\left(F_{1} \otimes \cdots \otimes F_{n}, W_{\lambda / \mu}^{(i)}\right), \operatorname{Ext}_{\mathcal{P}}^{*}\left(S_{\lambda / \mu}^{(i)}, F_{1} \otimes \cdots \otimes F_{n}\right)$

In the present paper we shall mainly deal with a very special case of the Decomposition Formula (already considered in [11]), namely the one for the diagram $\left(1^{d}\right)$. The Decomposition Formula in this case splits and takes the form of the well-known formula

$$
S^{d(i)}(V \oplus W)=\bigoplus_{j+k=d} S^{j(i)}(V) \otimes S^{k(i)}(W)
$$

Hence the Decomposition Spectral Sequence also splits and gives the formula:

$$
\operatorname{Ext}_{\mathcal{P}}^{*}\left(F_{1} \otimes \cdots \otimes F_{n}, S^{d(i)}\right)=\operatorname{Ext}_{\mathcal{P}}^{*}\left(F_{1}, S^{\left|F_{1}\right| / p^{i}(i)}\right) \otimes \cdots \otimes \operatorname{Ext}_{\mathcal{P}}^{*}\left(F_{n}, S^{\left|F_{n}\right| / p^{i}(i)}\right),
$$

for any homogeneous functors $F_{1}, \ldots, F_{n}$. We get analogous formulae for the divided and exterior powers and for products of homogeneous functors on the second variable instead of the first. The Schur functors for which the Decomposition Formula takes that simplest form were investigated in detail in [11] where they were called "exponential functors" (see [11, p. 670]). These particular instances of the Decomposition Formula and Decomposition Spectral Sequence will be referred to as the Exponential Formula. Some more advanced applications of the Decomposition Formula and Decomposition Spectral Sequence will appear in [6,7].

\section{Symmetrization of functor}

Let $\Sigma_{d}^{g r}$-mod denote the category of graded (by nonnegative integers) finitely generated in each degree $\mathbf{k}\left[\Sigma_{d}\right]$-modules and let $\mathbf{k}^{g r}$-mod denote the category of graded finite dimensional in each degree vector spaces over $\mathbf{k}$.

DEFINITION 3.1. - A functor $f: \Sigma_{d}^{g r}$-mod $\rightarrow \mathbf{k}^{g r}$-mod is called a $\Sigma_{d}$-functor if it satisfies two conditions:

(1) $f$ is k-linear. By this we mean that the structural map $\operatorname{Hom}_{\mathbf{k}\left[\Sigma_{d}\right]}(M, N) \rightarrow \operatorname{Hom}_{\mathbf{k}}(f(M)$, $f(N))$ is k-linear.

(2) $f$ commutes with forgetting the grading. This condition means that there exists a functor $\widetilde{f}: \Sigma_{d}$-mod $\rightarrow \mathbf{k}$-mod such that $Z \circ f=\tilde{f} \circ Z_{\Sigma}$ where $Z, Z_{\Sigma}$ are the functors of forgetting about grading in respective categories (such $\widetilde{f}$, if exists, is unique).

Let $f, g$ be $\Sigma_{d}$-functors. We call a transformation $\phi: f \rightarrow g$ a $\Sigma_{d}$-transformation if there exists a transformation $\widetilde{\phi}: \widetilde{f} \rightarrow \widetilde{g}$ such that $Z \circ \phi=\widetilde{\phi} \circ Z_{\Sigma}$.

We shall denote by $\mathcal{F}_{\Sigma_{d}}$ the category whose objects are $\Sigma_{d^{-}}$functors and morphisms are $\Sigma_{d^{-}}$ transformations.

Observe that for any $\Sigma_{d}$-functor $f$, an assignment $V \mapsto f\left(V^{\otimes d}\right)$ (we regard $V$ as concentrated in degree 0) defines a homogeneous strict polynomial functor of degree $d$ (we use here the first condition in the definition of $\Sigma_{d}$-functor). If so happens, we say that our $\Sigma_{d}$-functor $f$ 
is a symmetrization of the respective strict polynomial functor. In fact, we often define strict polynomial functors just giving their symmetrizations, e.g. $S^{d}=f\left(V^{\otimes d}\right)$, for the $\Sigma_{d}$-functor $f(M)=(M)_{\Sigma_{d}}$. Usually (if it causes no confusion) we will denote a symmetrization of a functor by the same letter but lower case. For example it is clear what we mean by $s^{\lambda}, d^{\lambda}, s_{\lambda}, w_{\lambda}$, e.g. $s_{\lambda}(M):=\operatorname{im}\left(\left(M^{\text {alt }}\right)^{\Sigma_{\lambda}} \rightarrow M \rightarrow(M)_{\Sigma_{\tilde{\lambda}}}\right.$ (for any $\Sigma_{d}$-module $M, M^{\text {alt }}$ stands for $M \otimes \operatorname{sgn}$ ). It is also self-evident how these functors behave with respect to the grading (degree of a tensor product is just a sum of degrees of factors) and that they commute with forgetting. One should be more cautious in the case of the exterior power for two reasons. The first is that the invariants and coinvariants of the alternating action are not isomorphic $\Sigma_{d}$-functors in general. So we should distinct between $\lambda_{\text {inv }}^{d}(M)=\left(M^{\text {alt }}\right)^{\Sigma_{d}}$, and $\lambda_{\text {coinv }}^{d}(M)=\left(M^{\text {alt }}\right)_{\Sigma_{d}}$, although both $\Sigma_{d}$-functors are symmetrizations of the strict polynomial functor $\Lambda^{d}$. The second reason is a pathology which happens for $p=2$, when we cannot define the exterior power as the (co)invariants of the alternating action. We will briefly discuss a modification which is needed in definition of $\lambda_{\text {inv }}^{d}$ (the argument for $\lambda_{\text {coinv }}^{d}$ is similar). We start with $d=2$. Then we may define $\lambda_{\text {inv }}^{2}$ as the kernel of the $\Sigma_{2}$-epimorphism id $\rightarrow s^{2}$. For an arbitrary $d$ we define $\lambda_{\text {inv }}^{d}$ to be $\bigcap_{\Sigma_{2} \subset \Sigma_{d}} \operatorname{ker}\left(\mathrm{id} \rightarrow \mathrm{id}_{\Sigma_{2}}\right)$. The definition meets our expectations because $\Sigma_{d}$ is generated by the set of transpositions. Its main advantage is that it refers only to a given action of the symmetric group. Therefore from now on we shall not consider the case $p=2$ separately.

It is worth mentioning that the idea of symmetrization is present in many constructions in representation theory. For example, applying certain $\Sigma_{d}$-functors to the $\Sigma_{d}$-bimodule $\mathbf{k}\left[\Sigma_{d}\right]$ we obtain some important $\Sigma_{d}$-modules (e.g. $s_{\lambda}\left(\mathbf{k}\left[\Sigma_{d}\right]\right)$ is the Specht module $\left.S p_{\lambda}\right)$. Finally, observe that we still have the Kuhn duality. Namely, for a $\Sigma_{d^{-}}$functor $f$, we put $f^{\#}(M):=\left(f\left(M^{*}\right)\right)^{*}$, where * at the right-hand side means the $\mathbf{k}$-linear duality. Now it is easy to see that $\left(s_{\lambda}\right)^{\#}=w_{\lambda}$ and in particular $\left(\lambda_{\text {inv }}^{d}\right) \#=\lambda_{\text {coinv }}^{d}$.

DEFINITION 3.2. - A $\Sigma_{d}$-functor $f^{\text {in }}$ is called an injective symmetrization of a functor $F \in \mathcal{P}_{d}$ if $f^{\text {in }}\left(V^{\otimes d}\right)=F(V)$, and there exists a $\Sigma_{d}$-transformation $\psi: f^{\text {in }} \rightarrow \bigoplus_{k} s^{\lambda^{k}}$ such that $\psi\left(V^{\otimes d}\right)$ is a monomorphism.

Similarly, we say that a symmetrization $f^{\mathrm{pr}}$ is a projective symmetrization if there exists a $\Sigma_{d}$-transformation $\phi: \bigoplus_{k} d^{\lambda^{k}} \rightarrow f^{\text {pr }}$ whose evaluation on $V^{\otimes d}$ is onto.

The importance of this class of symmetrizations comes from the fact that the family $\left\{S^{\lambda}\right\}$ (respectively $\left\{D^{\lambda}\right\}$ ) forms a set of injective (respectively projective) generators of $\mathcal{P}_{d}$ [13, Theorem 2.10]. In order to express concisely another important property of injective symmetrizations we need the following definition.

DEFINITION 3.3. - We say that a $\Sigma_{d}$-module $M$ is a $Y$-permutative module if $M \simeq$ $\bigoplus_{i=1}^{n} M_{i}$, where $M_{i}=\left(\mathbf{k} \otimes_{\mathbf{k}\left[H_{i}\right]} \mathbf{k}\left[\Sigma_{d}\right]\right)\left[j_{i}\right]\left(\left[j_{i}\right]\right.$ stands here for the shift of grading in a module which was originally placed in degree 0 ) for some Young subgroups $H_{i}$ in $\Sigma_{d}$ (cf. [15, Section 1.3]).

The most important example of a $Y$-permutative module is the $\Sigma_{d}$-module $V^{\otimes d}$ for any graded space $V$.

PROPOSITION 3.1.-

(1) Any strict polynomial functor has an injective and a projective symmetrization.

(2) Let $f^{\text {in }} \stackrel{\psi_{0}}{\rightarrow} s^{\lambda^{0}}$ be an injective symmetrization of $F$ (I adopt the convention: $s^{\lambda^{0}}:=$ $\left.\bigoplus_{k} s^{\lambda^{0 k}}\right)$. Then $\psi_{0}$ may be extended to a sequence of $\Sigma_{d}$-transformations

$$
f^{\text {in }} \stackrel{\psi_{0}}{\longrightarrow} s^{\lambda^{0}} \stackrel{\psi_{1}}{\longrightarrow} s^{\lambda^{1}} \stackrel{\psi_{2}}{\longrightarrow} \cdots \stackrel{\psi_{l}}{\longrightarrow} s^{\lambda^{l}},
$$

$4^{\mathrm{e}}$ SÉRIE - TOME $38-2005-\mathrm{N}^{\circ} 5$ 
such that for any $Y$-permutative $\Sigma_{d}$-module $M$, the sequence

$$
0 \rightarrow f^{\text {in }}(M) \stackrel{\psi_{0}}{\longrightarrow} s^{\lambda^{0}}(M) \stackrel{\psi_{1}}{\longrightarrow} s^{\lambda^{1}}(M) \stackrel{\psi_{2}}{\longrightarrow} \cdots \stackrel{\psi_{l}}{\longrightarrow} s^{\lambda^{l}}(M) \rightarrow 0
$$

is exact.

An analogous fact holds for a projective symmetrization.

Proof. - We start with comparing transformations and $\Sigma_{d}$-transformations in a very special case.

LEMMA 3.2. - For any diagrams $\lambda, \lambda^{\prime}$ of weight $d$

$$
\operatorname{Hom}_{\mathcal{P}_{d}}\left(S^{\lambda}, S^{\lambda^{\prime}}\right)=\operatorname{Hom}_{\mathcal{F}_{\Sigma_{d}}}\left(s^{\lambda}, s^{\lambda^{\prime}}\right) .
$$

Proof. - Since $\operatorname{Hom}_{\mathcal{P}}\left(S^{k}, S^{k}\right)=\mathbf{k}$, applying the Exponential Formula to both variables we get some description of $\operatorname{Hom}\left(S^{\lambda}, S^{\lambda^{\prime}}\right)$. From a purely combinatorial point of view we may describe it as a space having basis labeled by matrices consisting of positive integers satisfying the following conditions: each row is weakly decreasing, the sum of numbers in the $i$-th row equals $\lambda_{i}$, the sum of numbers in the $i$-th column equals $\lambda_{j}^{\prime}$ (cf. [11, Corollary 1.8]). Looking at the construction of the Decomposition Formula it is easy to find the transformation corresponding to a given element of the basis. Namely, to a matrix $\left[a_{i j}\right]$ we associate a composition

$$
S^{\lambda} \rightarrow \bigotimes_{i j} S^{a_{i j}} \simeq \bigotimes_{i j} S^{a_{j i}} \rightarrow S^{\lambda^{\prime}}
$$

where the first and third arrows are respectively tensor products of iterated comultiplication and multiplication in the symmetric power, while the second arrow interchanges factors which on the left-hand side are ordered with respect to rows and on the right-hand side with respect to columns (cf. [11, pp. 673-676]). Thus we see that any transformation is a composition of transformations of three simple types (possibly tensored with identities): the multiplication $S^{a} \otimes S^{b} \rightarrow S^{a+b}$, the comultiplication $S^{a+b} \rightarrow S^{a} \otimes S^{b}$, and the transposition $S^{a} \otimes S^{b} \rightarrow S^{b} \otimes S^{a}$. These transformations, of course, come from $\Sigma_{d}$-transformations, respectively from the induction, the restriction and the homomorphism of the groups. Thus we have shown that any transformation comes from some $\Sigma_{d}$-transformation.

It remains to show that a nontrivial $\Sigma_{d}$-transformation $\psi: s^{\lambda} \rightarrow s^{\lambda^{\prime}}$ has the nontrivial evaluation $\psi\left(V^{\otimes d}\right): S^{\lambda} \rightarrow S^{\lambda^{\prime}}$. It will be more convenient to work with the Kuhn dual of $\psi$, which is the $\Sigma_{d^{-}}$transformation $\psi^{\#}: d^{\lambda^{\prime}} \rightarrow d^{\lambda}$. Suppose that $\operatorname{im}\left(\psi^{\#}\right)\left(V^{\otimes d}\right)=0$. Then $\operatorname{im}\left(\psi^{\#}\right)$ is a left exact functor vanishing on all $\Sigma_{d}$-modules $V^{\otimes d}$. But if $\operatorname{dim}(V)=d$ then $V^{\otimes d}$ contains $\mathbf{k}\left[\Sigma_{d}\right]$ as a direct summand. Thus $\operatorname{im}\left(\psi^{\#}\right)\left(\mathbf{k}\left[\Sigma_{d}\right]\right)=0$. But since any finitely generated $\Sigma_{d^{-}}$ module embeds into a free module and $\operatorname{im}\left(\psi^{\#}\right)$ preserves monomorphisms, it must be the trivial functor.

In order to construct an injective symmetrization of a strict polynomial functor $F$ we consider the beginning of a finite injective resolution of $F$ by the sums of products of symmetric powers

$$
0 \rightarrow F \stackrel{\psi_{0}^{\prime}}{\longrightarrow} S^{\lambda^{0}} \stackrel{\psi_{1}^{\prime}}{\longrightarrow} S^{\lambda^{1}} \rightarrow \cdots
$$

The existence of such a finite resolution follows easily from the axioms for highest weight category [9, Definition 3.1] and the Littlewood-Richardson rule [3]. Of course $F=\operatorname{ker}\left(\psi_{1}^{\prime}\right)$. Thanks to Lemma 3.5 we know that the transformation $\psi_{1}^{\prime}$ comes from the $\Sigma_{d}$-transformation 
$\psi_{1}: s^{\lambda^{0}} \rightarrow s^{\lambda^{1}}$. Therefore the $\Sigma_{d}$-functor $f^{\text {in }}:=\operatorname{ker}\left(\psi_{1}\right)$ is an injective symmetrization of $F$, since it is obviously $\mathbf{k}$-linear and commutes with forgetting. This finishes the proof of the first part of Proposition 3.4.

To obtain the second part we take the whole resolution

$$
0 \rightarrow F \stackrel{\psi_{0}^{\prime}}{\longrightarrow} S^{\lambda^{0}} \stackrel{\psi_{1}^{\prime}}{\longrightarrow} S^{\lambda^{1}} \stackrel{\psi_{2}^{\prime}}{\longrightarrow} \cdots \stackrel{\psi_{l}^{\prime}}{\longrightarrow} S^{\lambda^{l}} \rightarrow 0 .
$$

According to Lemma 3.5 it lifts to the sequence of $\Sigma_{d}$-transformations

$$
0 \rightarrow f^{\text {in }} \stackrel{\psi_{0}}{\longrightarrow} s^{\lambda^{0}} \stackrel{\psi_{1}}{\longrightarrow} s^{\lambda^{1}} \stackrel{\psi_{2}}{\longrightarrow} \cdots \stackrel{\psi_{l}}{\longrightarrow} s^{\lambda^{l}} \rightarrow 0,
$$

whose evaluation on $V^{\otimes d}$ is exact. The exactness of evaluation on an arbitrary $Y$-permutative module follows from the fact, that any such a module is a direct summand in a finite sum of $V^{\otimes d}$ for a space $V$ of dimension $d$.

Of course, an injective symmetrization is not unique. The exterior power provides the easiest example of this, since both $\lambda_{\text {inv }}^{d} \rightarrow$ id and $\lambda_{\text {coinv }}^{d} \rightarrow$ id are injective symmetrizations of $\Lambda^{d}$. The point is that although the arrow $\lambda_{\text {coinv }}^{d} \rightarrow$ id ("averaging to invariants") is not monomorphic, its evaluation on $V^{\otimes d}$ is, which is sufficient. For a similar reason $s_{\lambda}$ is not only injective but also projective symmetrization of $S_{\lambda}$ (an analogous fact holds for Weyl functors).

We finish this section with one more tricky example of an injective symmetrization. We shall find an injective symmetrization of $S^{d(1)}$. To do this we consider the beginning of the de Rham complex (cf. [13, Theorem 4.1]) augmented by its 0 -th cohomology

$$
0 \rightarrow S^{d(1)} \rightarrow S^{p d} \stackrel{\delta}{\longrightarrow} S^{p d-1} \otimes \Lambda^{1}
$$

and we put $s^{d(1)}$ to be the kernel of the $\Sigma_{p d}$-transformation corresponding to the de Rham differential $\delta$. Note that $s^{d(1)}\left(V^{\otimes p d}\right)=S^{d(1)}(V)$ regarded as a graded space has degrees of nontrivial components multiplied by $p$ (we recall that the map $S^{d(1)} \rightarrow S^{p d}$ is given by the formula $\left.v_{1} \cdot \ldots \cdot v_{d} \mapsto v_{1}^{p} \cdot \ldots \cdot v_{d}^{p}\right)$.

It is also possible to describe explicitly an injective symmetrization of $S^{d(i)}$ for $i>1$, for Troesch [22] constructed a resolution of $S^{d(i)}$ by symmetric powers. We do not reproduce this resolution here, because it is quite complicated. We only warn the reader again that since the resolution extends the map $v_{1} \cdot \ldots \cdot v_{d} \mapsto v_{1}^{p^{i}} \cdot \ldots \cdot v_{d}^{p^{i}}$, the associated symmetrization multiplies degrees of nontrivial components by $p^{i}$. Taking into account these phenomena it is convenient to say that the Frobenius twist regarded as a functor on the graded spaces multiplies grading by $p$ (i.e. we put $V_{p i}^{(1)}:=V_{i}$ and 0 elsewhere). Taking this convention we may say that if $s^{d(i)}$ is an arbitrary injective symmetrization of $S^{d(i)}$ then $s^{d(i)}\left(V^{\otimes d p^{i}}\right)=S^{d(i)}(V)$ for any graded space $V$.

\section{The main theorems}

Let $A_{i}=\operatorname{Ext}^{*}\left(I^{(i)}, I^{(i)}\right), B_{i}=\left(A_{i}\right)^{\otimes d} \otimes \mathbf{k}\left[\Sigma_{d}\right]$ with a grading in $A_{i}$ coming from the grading on Ext-groups and the group algebra placed in degree 0 . We endow $B_{i}$ with a structure of $\Sigma_{d^{-}}$ bimodule given by the formula

$$
\sigma . a_{1} \otimes \cdots \otimes a_{d} \otimes e_{\tau} \cdot \lambda:=a_{\sigma(1)} \otimes \cdots \otimes a_{\sigma(d)} \otimes e_{\sigma \cdot \tau \cdot \lambda} .
$$

$4^{\text {e }}$ SÉRIE - TOME $38-2005-\mathrm{N}^{\circ} 5$ 
Sometimes it will be more convenient to look at $B_{i}$ as a bimodule with the action:

$$
\sigma . a_{1} \otimes \cdots \otimes a_{d} \otimes e_{\tau} \cdot \lambda=a_{\lambda^{-1}(1)} \otimes \cdots \otimes a_{\lambda^{-1}(d)} \otimes e_{\sigma \cdot \tau \cdot \lambda} .
$$

An isomorphism between these two structures is given by the map

$$
a_{1} \otimes \cdots \otimes a_{d} \otimes e_{\tau} \mapsto a_{\tau^{-1}(1)} \otimes \cdots \otimes a_{\tau^{-1}(d)} \otimes e_{\tau} .
$$

The main computational result of [13] was determination of $A_{i}$. It is a graded space which is one-dimensional in even degrees smaller than $2 p^{i}$ and trivial elsewhere. Now it follows easily from the Exponential Formula that

$$
\operatorname{Ext}^{*}\left(I^{d(i)}, I^{d(i)}\right)=B_{i},
$$

as a graded $\Sigma_{d}$-bimodule. It is also easy to see that the most important computations of [11, Section V] may be expressed in the form

$$
\operatorname{Ext}^{*}\left(F^{(i)}, G^{(i)}\right)=\left(g^{\text {in }}\left(f^{\text {pr\# }}\left(B_{i}\right)\right)\right),
$$

(i.e. we first apply $f^{\text {pr\# }}$ to $B_{i}$ as a left $\Sigma_{d}$-module and then we apply $g^{\text {in }}$ to the resulting right $\Sigma_{d}$-module). The main result of this section is determination of a class of functors for which this description holds.

We start with a slight generalization of the results of [11].

PROPOSITION 4.1.-

(1) For any diagrams $\mu, \mu^{\prime}, \lambda, \lambda^{\prime}$ of weight $d$ we have

$$
\operatorname{Ext}^{*}\left(D^{\mu(i)}, S^{\lambda(i)}\right)=s^{\mu}\left(s^{\lambda}\left(B_{i}\right)\right)=s^{\lambda}\left(s^{\mu}\left(B_{i}\right)\right),
$$

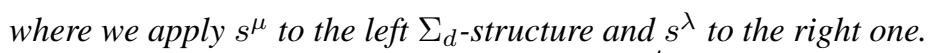

(2) Moreover, for any transformation $\psi: S^{\lambda} \rightarrow S^{\lambda^{\prime}}$ the induced map

$$
\psi_{*}^{(i)}: \operatorname{Ext}^{*}\left(D^{\mu(i)}, S^{\lambda(i)}\right) \rightarrow \operatorname{Ext}^{*}\left(D^{\mu(i)}, S^{\lambda^{\prime}(i)}\right)
$$

under the above isomorphisms, may be described in two ways: either as $\psi\left(s^{\mu}\left(B_{i}\right)\right)$ or as $s^{\mu}\left(\psi\left(B_{i}\right)\right)$. Similarly, for any transformation $\phi: D^{\mu} \rightarrow D^{\mu^{\prime}}$ the induced map $\phi^{(i) *}$ may be described either as $\phi^{\#}\left(s^{\lambda}\left(B_{i}\right)\right)$ or as $s^{\lambda}\left(\phi^{\#}\left(B_{i}\right)\right)$.

Proof. - The second description in the first part of the proposition for $\lambda=\mu=\left(1^{d}\right)$ is just [11, Theorem 4.5]. The general case follows from the Exponential Formula. The first description is the Kuhn dual of the second.

We now turn to the proof of the second part of Proposition 4.1 To get the first description we lift $\psi$ to some $\widetilde{\psi}: I^{d} \rightarrow I^{d}$ (the existence of such a lift follows from the projectivity of $I^{d}$ ) and consider the commutative diagram

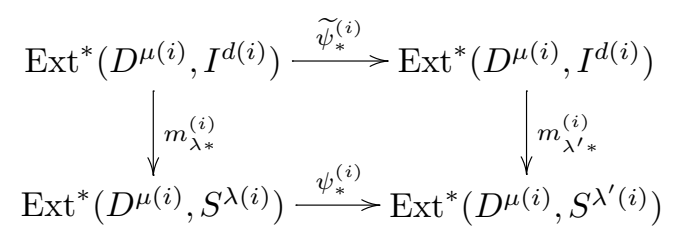


We recall from [11, Section V], that the vertical arrows are epimorphic and, according to the first part of the proposition, they may be identified respectively with $m_{\lambda}\left(s^{\mu}\left(B_{i}\right)\right)$ and $m_{\lambda^{\prime}}\left(s^{\mu}\left(B_{i}\right)\right)$. Moreover, since $\widetilde{\psi}$ is just multiplication by an element of $\mathbf{k}\left[\Sigma_{d}\right]$, we have $\widetilde{\psi}_{*}^{(i)}=\widetilde{\psi}\left(s^{\mu}\left(B_{i}\right)\right)$. Hence if we replace $\psi_{*}^{(i)}$ by $\psi\left(s^{\mu}\left(B_{i}\right)\right)$, the diagram remains commutative. But since the left vertical arrow is onto, there is at most one bottom arrow making the diagram commutative. Thus $\psi_{*}^{(i)}=\psi\left(s^{\mu}\left(B_{i}\right)\right)$.

In order to obtain the second description we consider the diagram

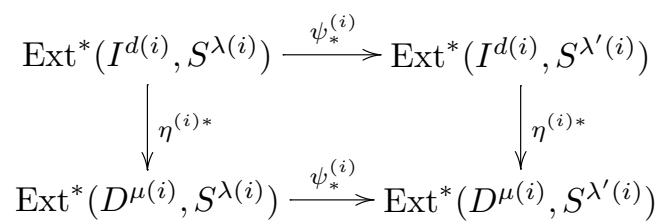

where $\eta_{\mu}: D^{\mu} \rightarrow I^{d}$ is the natural inclusion. After identifying known arrows we get

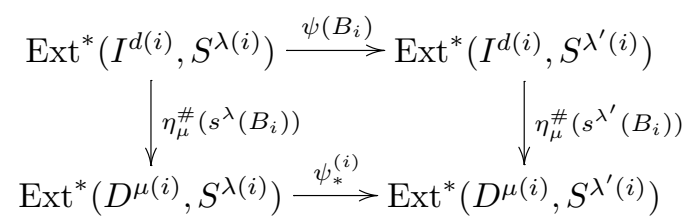

(for the vertical arrows we use a description which is Kuhn dual to that from [11] while for the top arrow we use the previous description for $\mu=\left(1^{d}\right)$ ). By the epimorphicity of the left vertical arrow it suffices to observe that $s^{\mu}\left(\psi\left(B_{i}\right)\right)$ makes the diagram commutative. The case of a transformation between divided powers follows from the Kuhn duality.

As it was seen in the proof, all the assertions of Proposition 4.1 were quite formal consequences of [11, Theorem 4.5] where the groups $\operatorname{Ext}^{*}\left(D^{d(i)}, S^{d(i)}\right)$ were computed. But this generalization, technically rather straightforward, will turn out to be extremely useful, for $\left\{D^{\lambda}\right\}$ (respectively $\left\{S^{\lambda}\right\}$ ) form a set of projective (respectively injective) generators of $\mathcal{P}_{d}$. Therefore our strategy for computing Ext-groups will be, roughly speaking, as follows. To compute $\operatorname{Ext}^{*}\left(F^{(i)}, G^{(i)}\right)$ we take a resolution of $F$ by (sums of products of) divided powers and a resolution of $G$ by symmetric powers, then we twist them $i$ times and we compute Extgroups between the (twisted) resolutions. By Proposition 4.1 we know these Ext-groups and also the arrows between them. This, under some additional hypotheses, will enable us to calculate the original Ext-groups.

For reasons apparent in the proof of Theorem 4.4, we also need an "additive analogue" of the last proposition. Put $A_{j}^{\prime}=\operatorname{Hom}(j I, I), B_{j}^{\prime}=\left(A_{j}^{\prime}\right)^{\otimes d} \otimes \mathbf{k}\left[\Sigma_{d}\right]$, where $j I$ denotes $I^{\oplus j}$. Thus, $A_{j}^{\prime}$ is just $j$-dimensional space concentrated in degree 0 .

PROPOSITION 4.2. -

(1) For any diagrams $\mu, \mu^{\prime}, \lambda, \lambda^{\prime}$ of weight $d$ we have

$$
\operatorname{Ext}^{*}\left(D^{\mu} \circ j I, S^{\lambda}\right)=s^{\mu}\left(s^{\lambda}\left(B_{j}^{\prime}\right)\right)=s^{\lambda}\left(s^{\mu}\left(B_{j}^{\prime}\right)\right),
$$

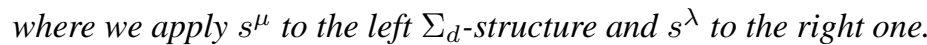

(2) Moreover, for any transformation $\psi: S^{\lambda} \rightarrow S^{\lambda^{\prime}}$ the induced map

$$
\psi_{*}: \operatorname{Ext}^{*}\left(D^{\mu} \circ j I, S^{\lambda}\right) \rightarrow \operatorname{Ext}^{*}\left(D^{\mu} \circ j I, S^{\lambda^{\prime}}\right)
$$

$4^{\text {e }}$ SÉRIE - TOME $38-2005-\mathrm{N}^{\circ} 5$ 
under the above isomorphisms, may be described in two ways: either as $\psi\left(s^{\mu}\left(B_{j}^{\prime}\right)\right)$ or as $s^{\mu}\left(\psi\left(B_{j}^{\prime}\right)\right)$. Similarly, for any transformation $\phi: D^{\mu} \rightarrow D^{\mu^{\prime}}$ the induced map $(\phi \circ j I)^{*}$ may be described either as $\phi^{\#}\left(s^{\lambda}\left(B_{j}^{\prime}\right)\right)$ or as $s^{\lambda}\left(\phi^{\#}\left(B_{j}^{\prime}\right)\right)$.

Proof. - First, observe that by the projectivity of $D^{\mu} \circ j I$ and the injectivity of $S^{\lambda}$ the map $s^{\mu}\left(s^{\lambda}\left(B_{j}^{\prime}\right)\right) \rightarrow \operatorname{Hom}\left(D^{\mu} \circ j I, S^{\lambda}\right)$ is an epimorphism. Hence, it suffices to show that both the spaces have the same dimensions. According to the Exponential Formula it suffices to do this for $\mu=\lambda=\left(1^{d}\right)$. Then $s^{d}\left(s^{d}\left(B_{j}^{\prime}\right)\right)=S^{d}\left(A_{j}^{\prime}\right)$, while

$$
\begin{aligned}
\operatorname{Hom}\left(D^{d} \circ j I, S^{d}\right) & =\bigoplus_{i_{1}+\cdots+i_{j}=d} \operatorname{Hom}\left(D^{i_{1}}, S^{i_{1}}\right) \otimes \cdots \otimes \operatorname{Hom}\left(D^{i_{j}}, S^{i_{j}}\right) \\
& =\bigoplus_{i_{1}+\cdots+i_{j}=d} S^{i_{1}}\left(A_{1}^{\prime}\right) \otimes \cdots \otimes S^{i_{j}}\left(A_{1}^{\prime}\right) .
\end{aligned}
$$

The dimensions of these spaces are clearly equal.

The proof of the second part goes in a similar fashion to that of the second part of Proposition 4.1. The only difference is that the epimorphicity of vertical arrows in the diagram

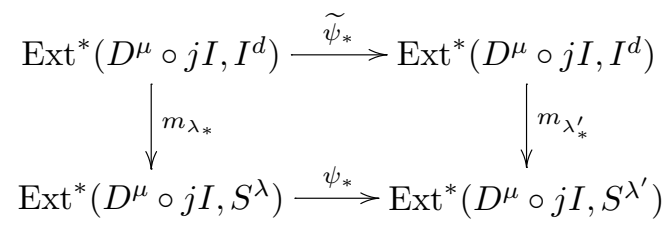

immediately follows from the projectivity of $D^{\mu} \circ j I$. This concludes the proof of Proposition 4.2 .

We are now in a position to state our first main result.

THEOREM 4.3.-

(1) For any $F \in \mathcal{P}_{d}$ and any diagram $\mu$ of weight $d$ :

$$
\operatorname{Ext}^{*}\left(D^{\mu(i)}, F^{(i)}\right)=f^{\text {in }}\left(s^{\mu}\left(B_{i}\right)\right),
$$

where $f^{\text {in }}$ is an arbitrary injective symmetrization of $F$.

(2) For any transformation $\phi: D^{\mu} \rightarrow D^{\mu^{\prime}}$, the induced map $\left(\phi^{(i) *}\right): \operatorname{Ext}^{*}\left(D^{\mu^{\prime}(i)}, F^{(i)}\right) \rightarrow$ $\operatorname{Ext}^{*}\left(D^{\mu(i)}, F^{(i)}\right)$, under the above isomorphisms takes the form $f^{\mathrm{in}}\left(\phi^{\#}\left(B_{i}\right)\right)$. Also "additive analogues" of these formulae hold, i.e.

$$
\operatorname{Hom}\left(D^{\mu} \circ j I, F\right)=f^{\text {in }}\left(s^{\mu}\left(B_{j}^{\prime}\right)\right),
$$

and $(\phi \circ j I)^{*}=f^{\text {in }}\left(\phi^{\#}\left(B_{j}^{\prime}\right)\right)$.

Proof. - We start by proving the additive version of the theorem. In order to get

$$
\operatorname{Hom}\left(D^{\mu} \circ j I, F\right)=f^{\text {in }}\left(s^{\mu}\left(B_{j}^{\prime}\right)\right),
$$

we extend the map $\psi_{0}\left(V^{\otimes d}\right): f^{\text {in }}\left(V^{\otimes d}\right) \rightarrow s^{\lambda^{0}}\left(V^{\otimes d}\right)$ to a resolution

$$
0 \rightarrow F \stackrel{\psi_{0}}{\longrightarrow} S^{\lambda^{0}} \stackrel{\psi_{1}}{\longrightarrow} S^{\lambda^{1}} \stackrel{\psi_{2}}{\longrightarrow} \cdots \stackrel{\psi_{l}}{\longrightarrow} S^{\lambda^{l}} \rightarrow 0
$$


(from now on we will slightly abuse notation denoting by the same letter a $\Sigma_{d}$-transformation and its evaluation on $\left.V^{\otimes d}\right)$. Since $\operatorname{Ext}^{n}\left(D^{\mu} \circ j I, F\right)=0$ for $n>0$, this complex remains exact after applying $\operatorname{Hom}\left(D^{\mu} \circ j I,-\right)$. We consider the diagram

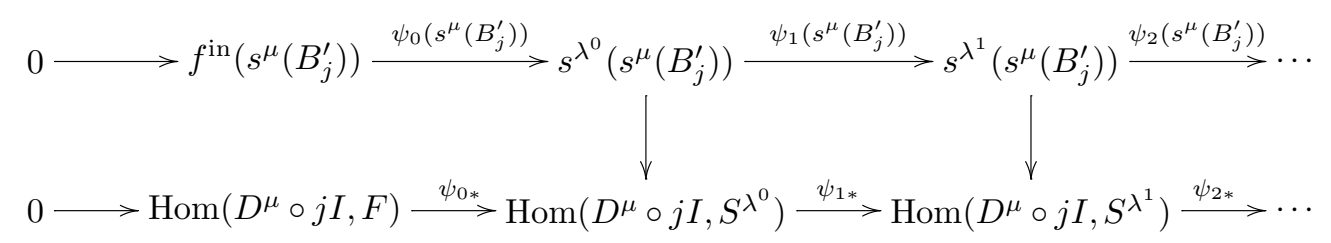

whose bottom row is exact. Note moreover, that thanks to Proposition 4.2, the vertical arrows (exist and) are isomorphisms and that the top row is exact by Proposition 3.4.2 $\left(s^{\mu}\left(B_{j}^{\prime}\right)\right.$ is a $Y$-permutative module because it is a tensor product of two $Y$-permutative modules). Thus, we get an isomorphism $f^{\text {in }}\left(s^{\mu}\left(B_{j}^{\prime}\right)\right) \simeq \operatorname{Hom}\left(D^{\mu} \circ j I, F\right)$ by an easy diagram chasing. We notice for future use that under this identification we have $\left(\psi_{0}\right)_{*}=\psi_{0}\left(s^{\mu}\left(B_{j}^{\prime}\right)\right)$. This is important because this time it need not to be true that $\psi_{0}\left(s^{\mu}\left(B_{j}^{\prime}\right)\right)=s^{\mu}\left(\psi_{0}\left(B_{j}^{\prime}\right)\right)$. The easiest example of this is provided by the arrow $\operatorname{Hom}\left(D^{p}, D^{p}\right) \rightarrow \operatorname{Hom}\left(D^{p}, I^{p}\right)$ induced by the inclusion $\psi_{0}: D^{p} \rightarrow I^{p}$ which may be thought of as the beginning of an injective resolution of $D^{p}$. Indeed: in this case $s^{p}\left(\psi_{0}\left(B_{1}^{\prime}\right)\right)$ is trivial. The existence of such phenomena will make us to be very careful in the further arguing.

We now turn to the second part of the additive version of the theorem. A transformation $\phi: D^{\mu} \rightarrow D^{\mu^{\prime}}$ induces a commutative diagram with exact rows

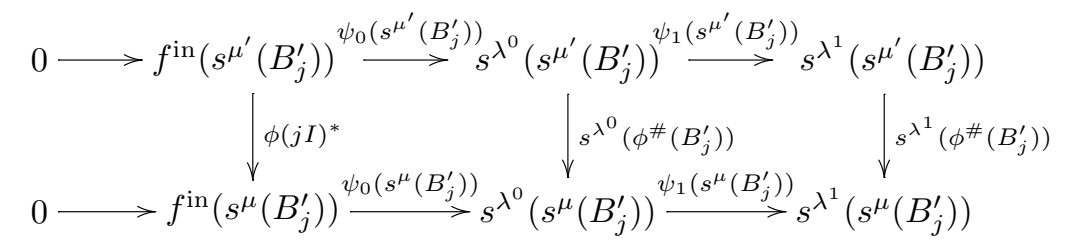

Now it suffices to observe that replacing $\phi(j I)^{*}$ by $f^{\text {in }}\left(\phi^{\#}\left(B_{j}^{\prime}\right)\right)$ does not destroy the commutativity of the diagram. It means that $\phi(j I)^{*}=f^{\text {in }}\left(\phi^{\#}\left(B_{j}^{\prime}\right)\right)$. Again it turns out that we could not take $\phi^{\#}\left(f^{\text {in }}\left(B_{j}^{\prime}\right)\right)$ instead of $f^{\text {in }}\left(\phi^{\#}\left(B_{j}^{\prime}\right)\right)$. A simple example of the arrow $\operatorname{Hom}\left(I^{p}, D^{p}\right) \rightarrow \operatorname{Hom}\left(D^{p}, D^{p}\right)$ induced by the inclusion $\phi: D^{p} \rightarrow I^{p}$ shows, that the maps $f^{\text {in }}\left(\phi^{\#}\left(B_{j}^{\prime}\right)\right)$ and $\phi^{\#}\left(f^{\text {in }}\left(B_{j}^{\prime}\right)\right)$ need not to coincide. It looks strange because, as we remember from Proposition 4.2, in all further vertical arrows they do coincide. But we recall that one cannot change the order of applying $\Sigma_{d}$-functors also on the left horizontal arrows.

We now turn to the proper version of Theorem 4.3. This time we first twist $i$ times an injective resolution of $F$ and then we apply to it $\operatorname{Ext}^{*}\left(D^{\mu(i)},-\right)$. According to Proposition 4.1 we get the commutative diagram

$$
\begin{aligned}
& 0 \longrightarrow f^{\text {in }}\left(s^{\mu}\left(B_{i}\right)\right) \stackrel{\psi_{0}\left(s^{\mu}\left(B_{i}\right)\right)}{\longrightarrow} s^{\lambda^{0}}\left(s^{\mu}\left(B_{i}\right)\right) \stackrel{\psi_{1}\left(s^{\mu}\left(B_{i}\right)\right)}{\longrightarrow} s^{\lambda^{1}}\left(s^{\mu}\left(B_{i}\right)\right) \stackrel{\psi_{2}\left(s^{\mu}\left(B_{i}\right)\right)}{\longrightarrow} \ldots \\
& 0 \longrightarrow \operatorname{Ext}^{*}\left(D^{\mu(i)}, F^{(i)}\right) \stackrel{\left(\psi_{0}^{(i)}\right)_{*}}{\longrightarrow} \operatorname{Ext}^{*}\left(D^{\mu(i)}, S^{\lambda^{0}(i)}\right) \stackrel{\left(\psi_{1}^{(i)}\right)_{*}}{\longrightarrow} \operatorname{Ext}^{*}\left(D^{\mu(i)}, S^{\lambda^{1}(i)}\right) \stackrel{\left(\psi_{2}^{(i)}\right)_{*}}{\longrightarrow} \cdots \\
& 4^{\mathrm{e}} \text { SÉRIE - TOME } 38-2005-\mathrm{N}^{\circ} 5
\end{aligned}
$$


in which all vertical arrows are isomorphisms. In order to finish the proof like in the additive version it is sufficient to show that the bottom row is exact. But we know that it is exact at least starting from the third term, because the top row is exact. It means that in the first hyperExt spectral sequence converging to

$$
\operatorname{hExt}^{*}\left(D^{\mu(i)}, \mathbf{C}\right)=0
$$

(where $\mathbf{C}$ stands for the twisted resolution of $F$ ), the $E^{2}$-term may be nontrivial only at the first two columns. Therefore, by dimension argument, it must be trivial (this argument generalizes $\mathbf{C}$ and may be easily derived from) a well known fact that if every third arrow in a long exact sequence is epimorphic then the sequence splits).

The proof of the second part is analogous to the proof of the additive counterpart. This completes the proof of Theorem 4.3

In order to repeat the argument with respect to the first variable we shall need an assumption guaranteeing the exactness of a complex in the situation when Proposition 3.4.2 is not applicable. Moreover, problems with functoriality make the formulation of the result more complicated and make us introduce another bit of notation. Let $0 \rightarrow G \stackrel{\psi_{0}}{\longrightarrow} S^{\lambda^{0}}$ be the beginning of an injective resolution of $G$ and $D^{\mu^{0}} \stackrel{\phi_{0}}{\longrightarrow} F \rightarrow 0$ be the beginning of a projective resolution of $F$. We consider the commutative diagram

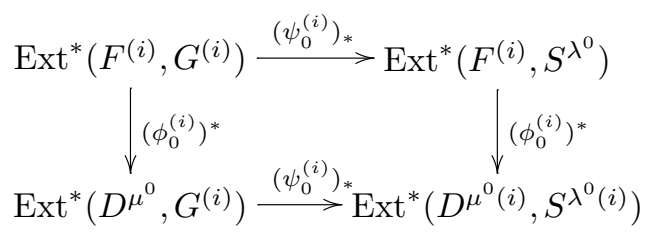

According to Theorem 4.3 we may rewrite it as

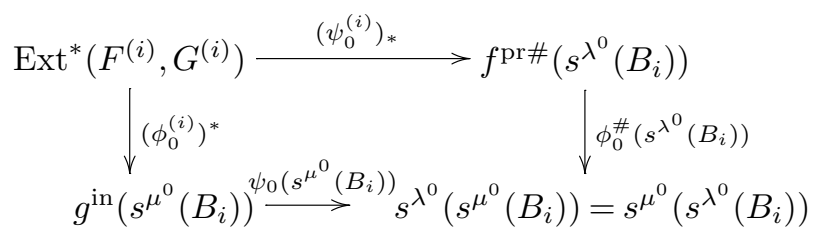

We put $\left(f^{\text {pr\# }}, g^{\text {in }}\right)\left(B_{i}\right)$ to be $\operatorname{im}\left(\psi_{0}\left(s^{\mu^{0}}\left(B_{i}\right)\right)\right) \cap \operatorname{im}\left(\phi_{0}^{\#}\left(s^{\lambda^{0}}\left(B_{i}\right)\right)\right)$. The point of this definition is that in general we cannot identify this space neither with $f^{\text {pr\# }}\left(g^{\text {in }}\left(B_{i}\right)\right)$ nor with $g^{\text {in }}\left(f^{\text {pr\# }}\left(B_{i}\right)\right)$. Nevertheless, this is certain explicitly defined space which is determined by the symmetrizations $f^{\text {in }}, g^{\mathrm{pr}}$. Quite naturally, this space will be our candidate for $\operatorname{Ext}^{*}\left(F^{(i)}, G^{(i)}\right)$ in general.

THEOREM 4.4. - Assume that $\operatorname{Ext}^{*}\left(F \circ p^{i} I, G\right)=0$ for $*>0$ (we call this assumption the “Ext-condition”). Then

$$
\operatorname{Ext}^{*}\left(F^{(i)}, G^{(i)}\right)=\left(f^{\text {pr\# }}, g^{\text {in }}\right)\left(B_{i}\right)
$$

Proof. - We take an injective resolution of $F$, a projective resolution of $G$ and consider the diagram 


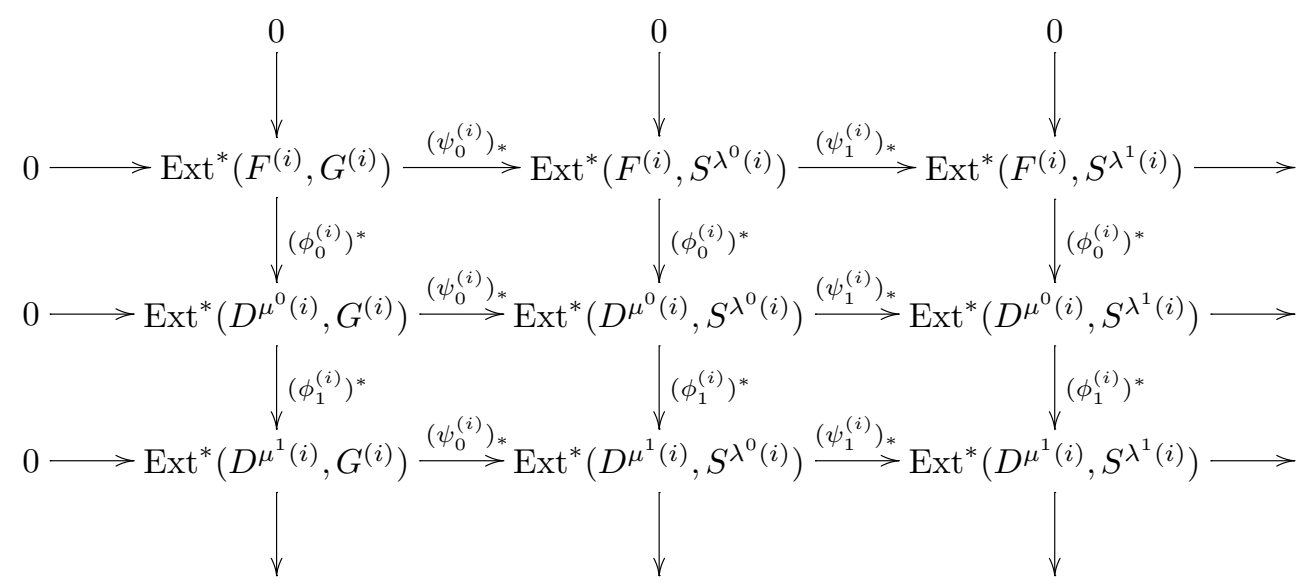

According to Theorem 4.3 all the rows except perhaps the first and all the columns except perhaps the first are exact. Hence, the proof will be finished by a diagram chasing if we show that the first column is exact. By Theorem 4.3 we have in the first column the sequence

$$
0 \rightarrow \operatorname{Ext}^{*}\left(F^{(i)}, G^{(i)}\right) \stackrel{\left(\phi_{0}^{(i)}\right)^{*}}{\longrightarrow} g^{\text {in }}\left(s^{\mu^{0}}\left(B_{i}\right)\right) \stackrel{\phi_{1}^{\#}\left(g^{\text {in }}\left(B_{i}\right)\right)}{\longrightarrow} g^{\text {in }}\left(s^{\mu^{1}}\left(B_{i}\right)\right) \stackrel{\phi_{2}^{\#}\left(g^{\text {in }}\left(B_{i}\right)\right)}{\longrightarrow} .
$$

Now we consider the sequence

$$
\cdots \stackrel{\phi_{2}}{\longrightarrow} D^{\mu^{1}} \circ p^{i} I \stackrel{\phi_{1}\left(p^{i} I\right)}{\longrightarrow} D^{\mu^{0}} \circ p^{i} I \stackrel{\phi_{0}\left(p^{i} I\right)}{\longrightarrow} F \circ p^{i} I \rightarrow 0 .
$$

Since it is a projective resolution of $F \circ p^{i} I$ and $\operatorname{Ext}^{n}\left(F \circ p^{i} I, G\right)=0$ for $n>0$, the sequence

$0 \rightarrow \operatorname{Hom}\left(F \circ p^{i} I, G\right) \stackrel{\left(\phi_{0}\left(p^{i} I\right)\right)^{*}}{\longrightarrow} \operatorname{Hom}\left(D^{\mu^{0}} \circ p^{i} I, G\right) \stackrel{\left(\phi_{1}^{\#}\left(p^{i} I\right)\right)^{*}}{\longrightarrow} \operatorname{Hom}\left(D^{\mu^{1}} \circ p^{i} I, G\right) \stackrel{\left(\phi_{2}^{\#}\left(p^{i} I\right)\right)^{*}}{\longrightarrow}$

is exact. But thanks to Theorem 4.3 we may rewrite it as

$$
0 \rightarrow \operatorname{Hom}\left(F \circ p^{i} I, G\right) \stackrel{\left(\phi_{0}\left(p^{i} I\right)\right)^{*}}{\longrightarrow} g^{\mathrm{in}}\left(s^{\mu^{0}}\left(B_{p^{i}}^{\prime}\right)\right) \stackrel{\phi_{1}^{\#}\left(g^{\mathrm{in}}\left(B_{p^{i}}^{\prime}\right)\right)}{\longrightarrow} g^{\text {in }}\left(s^{\mu^{1}}\left(B_{p^{i}}^{\prime}\right)\right) \stackrel{\phi_{2}^{\#}\left(g^{\mathrm{in}}\left(B_{p^{i}}^{\prime}\right)\right)}{\longrightarrow} .
$$

Let us now compare the above sequence with the sequence $(*)$. We see that, starting from the second term, the same $\Sigma_{d}$-functors and $\Sigma_{d}$-transformations appear in both sequences. The only difference is that in the first sequence we apply them to the graded $\Sigma_{d}$ bimodule $B_{i}$ while in the second to $B_{p^{i}}^{\prime}$. But since $\operatorname{dim}\left(A_{i}\right)=\operatorname{dim}\left(A_{p^{i}}^{\prime}\right), B_{i}$ and $B_{p^{i}}^{\prime}$ become isomorphic after forgetting about grading. Hence, since $\Sigma_{d}$-functors and $\Sigma_{d}$-transformations commute with forgetting, the exactness of $(*)$ gives us the exactness of our first column starting from the third term (in order to use this argument we have introduced all these "additive analogues"). Then we apply the hyperExt-argument which we used at the end of the proof of Theorem 4.3 to conclude that the whole column is exact. This completes the proof of Theorem 4.4.

\section{Reformulation and some special cases of Theorem 4.3}

All proofs in the previous section depended heavily on the notion of injective symmetrization. But finding an injective symmetrization of a given functor may be difficult. For this reason in this section we shall restate Theorem 4.3 in a way which does not refer to symmetrizations.

$4^{\mathrm{e}}$ SÉRIE - TOME $38-2005-\mathrm{N}^{\circ} 5$ 
For a functor $F \in \mathcal{P}_{d}$ and a Young diagram $\lambda=\left(\lambda_{1}, \ldots, \lambda_{k}\right)$ we define $\widetilde{F}^{\lambda}$ to be the summand of multidegree $\left(\lambda_{1}, \ldots, \lambda_{k}\right)$ in the $d$-functor $\left(V_{1}, \ldots, V_{k}\right) \mapsto F\left(V_{1} \oplus \cdots \oplus V_{k}\right)$. Then we put $F^{\lambda}(V):=\widetilde{F}^{\lambda}(V, \ldots, V)$.

COROLlARY 5.1. - For any $F \in \mathcal{P}_{d}$ and any diagram $\lambda$ of weight $d$, there is a vector space isomorphism:

$$
\operatorname{Ext}^{*}\left(D^{\lambda(i)}, F^{(i)}\right) \simeq F^{\lambda}\left(A_{i}\right) .
$$

Proof. - Our reformulation follows easily from the lemma which allows us to express the $\Sigma_{d^{-}}$ module $s^{\lambda}\left(B_{i}\right)$ in terms of weight spaces.

LEMMA 5.2. - There is a $\Sigma_{d}$-invariant isomorphism of functors:

$$
I^{d} \otimes_{\Sigma_{\lambda}} \mathbf{k}\left[\Sigma_{d}\right] \simeq\left(I^{d}\right)^{\lambda}
$$

Proof. - We identify $\left(I^{d}\right)^{\lambda}(V)$ with a subspace of $\left(\bigoplus_{j=1}^{k} V_{j}\right)^{\otimes d}$ spanned by elementary tensors having $\lambda_{j}$ factors belonging to $V_{j}$. Let $v^{j}$ for $v \in V$ indicate that we regard $v$ as an element of $V_{j}$. Then the map:

$$
v_{1} \otimes \cdots \otimes v_{d} \otimes \sigma \mapsto v_{\sigma(1)}^{1} \otimes \cdots \otimes v_{\sigma\left(\lambda_{1}\right)}^{1} \otimes v_{\sigma\left(\lambda_{1}+1\right)}^{2} \otimes \cdots \otimes v_{\sigma(d)}^{k}
$$

is the required isomorphism.

Therefore, putting $V=A_{i}$ in Lemma 5.2 we get an isomorphism of $\Sigma_{d}$-modules:

$$
s^{\lambda}\left(B_{i}\right)=A_{i}^{\otimes d} \otimes_{\Sigma_{\lambda}} \mathbf{k}\left[\Sigma_{d}\right] \simeq\left(I^{d}\right)^{\lambda}\left(A_{i}\right) .
$$

Therefore we have:

$$
\operatorname{Ext}^{*}\left(D^{\lambda(i)}, F^{(i)}\right)=f^{\text {in }}\left(s^{\lambda}\left(B_{i}\right)\right) \simeq f^{\text {in }}\left(\left(I^{d}\right)^{\lambda}\left(A_{i}\right)\right)=\left(f^{\text {in }} \circ I^{d}\right)^{\lambda}\left(A_{i}\right)=F^{\lambda}\left(A_{i}\right) .
$$

Remark. - The above formula is of course much more explicit than that appearing in Theorem 4.3. The only problem is that it, as stated, does not tell us anything about Ext-grading. If we want to derive information about grading from this formula, then some additional work is required. Namely, we should extend $F$ to a functor on graded spaces in a way which is compatible with a symmetrization. By this we mean that the isomorphism $F(V) \simeq f^{\text {in }}\left(V^{\otimes d}\right)$ preserves grading.

Let us now look at our formula in some special cases. For $\lambda=(d)$ it simplifies to the form

COROLlARY 5.3. - For any $F \in \mathcal{P}_{d}$, there is a vector space isomorphism:

$$
\operatorname{Ext}^{*}\left(D^{d(i)}, F^{(i)}\right) \simeq F\left(A_{i}\right) .
$$

We shall illustrate the above formula and our remark concerning grading by a simple example. For $F=I^{(j)}$ we get $\operatorname{Ext}^{*}\left(D^{p^{j}(i)}, I^{(i+j)}\right) \simeq A_{i}^{(j)}$. But we recall that the functor $I^{(j)}$ extended to the graded spaces multiplies degrees of nontrivial components by $p^{j}$ (see the discussion at the end of Section 3). Taking this into account we get the result predicted by [13, Theorem 4.5].

For the diagram $\lambda=\left(1^{d}\right)$, our formula:

$$
\operatorname{Ext}^{*}\left(I^{d(i)}, F^{(i)}\right) \simeq F^{\left(1^{d}\right)}\left(A_{i}\right)
$$


can also be obtained directly by using the Decomposition Formula. But this time it is interesting to compare the formulae from Theorem 4.3 and Corollary 5.1 for $i=0$. Since $\operatorname{dim}\left(A_{0}\right)=1$, we get an isomorphism of $\Sigma_{d}$-modules:

$$
f^{\mathrm{in}}\left(\mathbf{k}\left[\Sigma_{d}\right]\right) \simeq F^{\left(1^{d}\right)}(\mathbf{k}) .
$$

The right-hand side regarded as a functor in $F$ can be used to compare strict polynomial functors and $\mathbf{k}\left[\Sigma_{d}\right]$-modules. For example it was shown in [20, Section A4] that for $\mathbf{k}$ of characteristic 0 , the assignment $F \mapsto F^{\left(1^{d}\right)}(\mathbf{k})$ provides an equivalence between $\mathcal{P}_{d}$ and the category of finitely generated $\mathbf{k}\left[\Sigma_{d}\right]$-modules. Our formula offers some explanation of this equivalence, for it says that the functor $F$ and the $\Sigma_{d}$-module $F^{\left(1^{d}\right)}(\mathbf{k})$ are obtained by applying the same $\Sigma_{d}$-functor $f^{\text {in }}$ to the right $\Sigma_{d}$-structure of: $V^{\otimes d}$ in the first case, and $\mathbf{k}\left[\Sigma_{d}\right]$ in the second.

We finish this section by considering Corollary 5.1 for $i=0$ and an arbitrary $\lambda$. Then, for $D^{\lambda}$ is projective, we obtain:

$$
\operatorname{Hom}\left(D^{\lambda}, F\right) \simeq F^{\lambda}\left(A_{0}\right),
$$

which is nothing but [13, Corollary 2.12] expressed in a slightly more invariant way.

\section{Some special cases of Theorem 4.4}

The aim of this section is to find some functors which satisfy the assumptions of Theorem 4.4. The most important example is provided by $F=W_{\mu / \mu^{\prime}}$ and $G=S_{\lambda / \lambda^{\prime}}$. Indeed, Fact 2.1 together with the Decomposition Formula show that they satisfy the Ext-condition. Moreover, in this particular case the statement of the theorem may be formulated in a much simpler way.

THEOREM 6.1. - For any skew diagrams $\mu / \mu^{\prime}, \lambda / \lambda^{\prime}$ of weight $d$ we have

$$
\operatorname{Ext}^{*}\left(W_{\mu / \mu^{\prime}}^{(i)}, S_{\lambda / \lambda^{\prime}}^{(i)}\right)=s_{\mu / \mu^{\prime}}\left(s_{\lambda / \lambda^{\prime}}\left(B_{i}\right)\right)=s_{\lambda / \lambda^{\prime}}\left(s_{\mu / \mu^{\prime}}\left(B_{i}\right)\right) .
$$

Moreover, for any transformation $\psi: s_{\lambda / \lambda^{\prime}} \rightarrow s_{\lambda^{1} / \lambda^{1^{\prime}}}$ the induced map

$$
\psi_{*}: \operatorname{Ext}^{*}\left(W_{\mu / \mu^{\prime}}^{(i)}, S_{\lambda / \lambda^{\prime}}^{(i)}\right) \rightarrow \operatorname{Ext}^{*}\left(W_{\mu / \mu^{\prime}}^{(i)}, S_{\lambda^{1} / \lambda^{1^{\prime}}}^{(i)}\right)
$$

takes the form $\psi\left(s_{\mu / \mu^{\prime}}\left(B_{i}\right)\right)=s_{\mu / \mu^{\prime}}\left(\psi\left(B_{i}\right)\right)$. An analogous fact also holds for transformations of the first variable.

Proof. - When we look once again at the proof of Theorem 4.4, we see that the reason for which we could not obtain a simpler description of the Ext-groups was that in general the map $\psi: s^{\lambda} \rightarrow s^{\lambda^{\prime}}$ induces on $\operatorname{Ext}^{*}\left(F^{(i)},-\right)$ the map $f^{\text {in\# }}\left(\psi\left(B_{i}\right)\right)$ which may be different from the map $\psi\left(f^{\text {in\# }}\left(B_{i}\right)\right)$. We will show that for $F=W_{\mu / \mu^{\prime}}$ and $G=S_{\lambda / \lambda^{\prime}}$ these two maps coincide. By arguments used in the proof of the second part of Proposition 4.1 it suffices to show the lemma (which is very specific to Weyl and Schur functors):

LEMMA 6.2. - For any diagrams $\mu / \mu^{\prime}$ and $\lambda$, the map $m_{\lambda}: \mathrm{id} \rightarrow s^{\lambda}$ induces an epimorphism $\operatorname{Ext}^{*}\left(W_{\mu / \mu^{\prime}}^{(i)}, I^{d(i)}\right) \rightarrow \operatorname{Ext}^{*}\left(W_{\mu / \mu^{\prime}}^{(i)}, S^{\lambda(i)}\right)$.

Proof. - Of course, it suffices to show the additive version of the lemma. Applying the Decomposition Formula to $W_{\mu / \mu^{\prime}} \circ p^{i} I$, we reduce the proof to showing that there is an epimorphism $\operatorname{Hom}\left(W_{\mu / \mu^{\prime}}, I^{d}\right) \rightarrow \operatorname{Hom}\left(W_{\mu / \mu^{\prime}}, S^{\lambda}\right)$. To do this it suffices to show that 
$\operatorname{Ext}^{1}\left(W_{\mu / \mu^{\prime}}, \operatorname{ker}\left(m_{\lambda}\right)\right)=0$. By the Littlewood-Richardson rule [3], any skew Schur functor has a filtration with a graded object being a sum of Schur functors. Thus it suffices to establish the last formula for $\mu^{\prime}=\emptyset$. Using the Littlewood-Richardson rule again, we observe that the structural inclusion $W_{\mu} \rightarrow \Lambda^{\mu}$ has a cokernel with a graded object being a sum of Schur functors for diagrams lexicographically smaller than $\mu$ (the reason is that $\mu$ is the largest diagram appearing in the Littlewood-Richardson decomposition of $\Lambda^{\mu}$ ). Thus using induction on the lexicographic ordering we reduce our task to showing that $\operatorname{Ext}^{1}\left(\Lambda^{\mu}, \operatorname{ker}\left(m_{\lambda}\right)\right)=0$. By the Decomposition Formula, the last statement is equivalent to the fact that $m_{\lambda}$ induces an epimorphism $\operatorname{Hom}\left(\Lambda^{d}, I^{d}\right) \rightarrow \operatorname{Hom}\left(\Lambda^{d}, S^{\lambda}\right)$, which is clear by Fact 2.1. This completes the proof of the lemma.

Therefore we may choose the more convenient order of applying $\Sigma_{d}$-functors in the main diagram in the proof of Theorem 4.4. In particular, we may identify the morphism $\left(\psi_{\lambda / \lambda^{\prime}}^{(i)}\right)_{*}: \operatorname{Ext}^{*}\left(W_{\mu / \mu^{\prime}}^{(i)}, S_{\lambda / \lambda^{\prime}}^{(i)}\right) \rightarrow \operatorname{Ext}^{*}\left(W_{\mu / \mu^{\prime}}^{(i)}, S^{\overline{\lambda / \lambda^{\prime}}(i)}\right)$ with the map $\psi_{\lambda / \lambda^{\prime}}\left(s_{\mu / \mu^{\prime}}\left(B_{i}\right)\right)=$ $s_{\mu / \mu^{\prime}}\left(\psi_{\lambda / \lambda^{\prime}}\left(B_{i}\right)\right)$. This enables us to identify the Ext-groups with $s_{\mu / \mu^{\prime}}\left(s_{\lambda / \lambda^{\prime}}\left(B_{i}\right)\right)$ or $s_{\lambda / \lambda^{\prime}}\left(s_{\mu / \mu^{\prime}}\left(B_{i}\right)\right)$. In a similar fashion we obtain the desired description of induced maps. The second description of Ext-groups is the Kuhn dual of the first.

In particular for $\mu=\left(1^{d}\right)$ and $\mu=(d)$ we get respectively

$$
\operatorname{Ext}^{*}\left(D^{d(i)}, S_{\lambda / \lambda^{\prime}}^{(i)}\right)=s_{\lambda / \lambda^{\prime}}\left(s^{d}\left(B_{i}\right)\right)=s_{\lambda / \lambda^{\prime}}\left(A_{i}^{\otimes d}\right)=S_{\lambda / \lambda^{\prime}}\left(A_{i}\right),
$$

and

$$
\operatorname{Ext}^{*}\left(\Lambda^{d(i)}, S_{\lambda / \lambda^{\prime}}^{(i)}\right)=s_{\lambda / \lambda^{\prime}}\left(\lambda_{\text {coinv }}^{d}\left(B_{i}\right)\right)=s_{\lambda / \lambda^{\prime}}\left(\left(A_{i}^{\otimes d}\right)^{\text {alt }}\right)=W_{\widetilde{\lambda / \lambda^{\prime}}}\left(A_{i}\right),
$$

which generalizes computations of [11, Section V]. The only computation in [11] among those concerning diagrams of the same weight (we deal with diagrams of different weights in [7]) which does not fit this scheme is a computation of $\operatorname{Ext}^{*}\left(D^{d(i)}, D^{d(i)}\right)$. But these (and more general) groups may be computed directly from our Corollary 5.1. Indeed, it yields the formula

$$
\operatorname{Ext}^{*}\left(D^{d(i)}, W_{\lambda / \lambda^{\prime}}^{(i)}\right)=W_{\lambda / \lambda^{\prime}}\left(A_{i}\right) .
$$

On the other hand, one should be cautious using Theorem 6.1. For example, it is easy to see that the epimorphism $\psi: I^{p} \rightarrow \Lambda^{p}$ induces the trivial map $\operatorname{Hom}\left(\Lambda^{p}, I^{p}\right) \rightarrow \operatorname{Hom}\left(\Lambda^{p}, \Lambda^{p}\right)$ which seems to contradict Theorem 6.1 which says that it should be an epimorphism. The point is that we should consider the second variable as a Schur functor so its appropriate symmetrization is $\lambda_{\text {inv }}^{p}$ (we cannot take an arbitrary injective symmetrization in Theorem 6.1). Thus the corresponding $\Sigma_{d}$-transformation $\widetilde{\psi}$ : id $\rightarrow \lambda_{\text {inv }}^{p}$ is the averaging to invariants which is not an epimorphism in general.

Let us now try and look for other functors satisfying the assumption of Theorem 4.4. Taking into account Fact 2.2, it is tempting to consider Schur functors $S_{\mu}, S_{\lambda}$ satisfying $\lambda \not \mu$, since we have $\operatorname{Ext}^{*}\left(S_{\mu}, S_{\lambda}\right)=0$ for $*>0$. But in fact, we need the stronger condition: $\operatorname{Ext}^{*}\left(S_{\mu} \circ j I, S_{\lambda}\right)=0$ for $j=p^{i}$. When we apply the Decomposition Formula to $S_{\mu} \circ j I$ we see that our lexicographic assumption is quickly weakening. A counterexample is very simple: already for $\mu=\lambda=\left(2^{2}\right), p=2$ we get $\operatorname{Ext}^{2}\left(S_{\mu} \circ 2 I, S_{\lambda}\right) \neq 0$. A pathological element comes from the decomposition of $\mu$ into $\left(1^{2}\right),\left(1^{2}\right)$ and $\lambda$ into (2), (2). Also as small lexicographically diagram as $(2 k-1,1)$ and as large as $\left(2^{k}\right)$ may be decomposed to give a nontrivial element in $\operatorname{Ext}^{*}\left(S_{(2 k-1,1)} \circ 2 I, S_{\left(2^{k}\right)}\right)$ for $p=2$. Slightly more complicated examples can be constructed for $p>2$, and also for diagrams of very special shapes e.g. for "hooks". The only quite general 
class of Schur functors satisfying the assumption of Theorem 4.4 is provided by the diagrams of weight $p$.

COROLLARY 6.3. - If $\lambda \not \mu$ are diagrams of weight $p$ then

$$
\operatorname{Ext}^{*}\left(S_{\mu}^{(i)}, S_{\lambda}^{(i)}\right)=\left(w_{\mu}, s_{\lambda}\right)\left(B_{i}\right)
$$

Proof. - Observe that when we decompose $\mu$ into smaller diagrams we get diagrams of weight smaller than $p$ for which Schur functors are projective. This together with the lexicographic assumption gives the Ext-condition.

In the above case there is no reason for expecting that the formula will simplify to a form similar to that of Theorem 6.1. The general formula from Theorem 4.4 is not very convenient in practice. In order to rephrase the result in a more explicite form, we shall need one easy general fact.

LEMMA 6.4. - Let $0 \rightarrow F_{1} \rightarrow \cdots \rightarrow F_{k} \rightarrow 0$ be an exact sequence whose all objects satisfy the Ext-condition with some $G$. Then the sequence $0 \rightarrow \operatorname{Ext}^{*}\left(F_{k}^{(i)}, G^{(i)}\right) \rightarrow \cdots \rightarrow$ $\operatorname{Ext}^{*}\left(F_{1}^{(i)}, G^{(i)}\right) \rightarrow 0$ is exact.

Proof. - The assertion follows immediately from the fact that, according to Theorem 4.4, all Ext-groups under consideration are concentrated in even degrees.

Again it seems that the last result is in conflict with the fact that $\psi: I^{p} \rightarrow \Lambda^{p}$ induces the trivial map $\operatorname{Hom}\left(\Lambda^{p}, I^{p}\right) \rightarrow \operatorname{Hom}\left(\Lambda^{p}, \Lambda^{p}\right)$. But the point is that $\psi$ cannot be extended to an exact sequence satisfying the Ext-condition with $D^{d}$.

Now we would like to obtain a more explicit description of Ext-groups appearing in Corollary 6.3. To this end, we shall need a resolution of $S_{\mu}$ by exterior powers starting with the structural arrow $\phi_{\mu}$. The existence of such a resolution may be derived from some corollary of Theorem 6.1 and Lemma 6.4.

COROLLARY 6.5. - For any resolution $0 \rightarrow S_{\lambda} \stackrel{\psi_{\lambda}}{\longrightarrow} S^{\widetilde{\lambda}} \rightarrow \cdots$, there exists a "Koszul dual complex" $0 \rightarrow W_{\widetilde{\lambda}} \stackrel{\phi_{\lambda}^{\#}}{\longrightarrow} \Lambda^{\widetilde{\lambda}} \rightarrow \cdots$, which is exact (for explanation of this phenomenon see [8]).

Proof. - We apply the functor $\operatorname{Ext}^{*}\left(\Lambda^{d(i)},-\right)$ to the complex $0 \rightarrow S_{\lambda}^{(i)} \stackrel{\psi_{\lambda}}{\longrightarrow} S^{\widetilde{\lambda}(i)} \rightarrow \cdots$. According to Theorem 6.1 we get the complex $0 \rightarrow W_{\tilde{\lambda}}\left(A_{i}\right) \stackrel{\phi_{\bar{\lambda}}^{\#}\left(A_{i}\right)}{\longrightarrow} \Lambda^{\widetilde{\lambda}}\left(A_{i}\right) \rightarrow \cdots$, whose exactness follows from Lemma 6.4. Since the dimension of $A_{i}$ may be arbitrarily large, the whole complex of functors must be exact.

To obtain the desired resolution of $S_{\mu}$ by exterior powers, we take the Kuhn dual of the complex $0 \rightarrow W_{\mu} \stackrel{\phi_{\mu}^{\#}}{\longrightarrow} \Lambda^{\mu} \rightarrow \cdots$ constructed in Corollary 6.5 (for $\lambda:=\widetilde{\mu}$ ). Thus we get the resolution

$$
\cdots \rightarrow \Lambda^{\mu^{1}} \stackrel{\phi_{1}}{\longrightarrow} \Lambda^{\mu} \stackrel{\phi_{\mu}}{\longrightarrow} S_{\mu} \rightarrow 0 .
$$

Now observe that since exterior powers are also Weyl functors, they satisfy the Ext-condition with $S_{\lambda}$. Since $S_{\mu}$ satisfies it too, the sequence

$$
0 \rightarrow \operatorname{Ext}^{*}\left(S_{\mu}^{(i)}, S_{\lambda}^{(i)}\right) \stackrel{\left(\phi_{\mu}\right)^{*}}{\longrightarrow} \operatorname{Ext}^{*}\left(\Lambda^{\mu(i)}, S_{\lambda}^{(i)}\right) \stackrel{\left(\phi_{1}\right)^{*}}{\longrightarrow} \cdots
$$

$4^{\text {e }}$ SÉRIE - TOME $38-2005-\mathrm{N}^{\circ} 5$ 
is exact by Lemma 6.4. Hence $\operatorname{Ext}^{*}\left(\Lambda^{\mu(i)}, S_{\lambda}^{(i)}\right)=\operatorname{ker}\left(\left(\phi_{1}\right)^{*}\right)$. In order to compute this kernel we observe that all groups and arrows appearing in the above sequence starting from the second term are known by Theorem 6.1: $\operatorname{Ext}^{*}\left(\Lambda^{\mu(i)}, S_{\lambda}^{(i)}\right)=\lambda_{i}^{\mu}\left(s_{\lambda}\left(B_{i}\right)\right)$ etc. Let $\widetilde{\phi}_{1}: \lambda_{c}^{\mu^{1}} \rightarrow \lambda_{c}^{\mu}$ be the $\Sigma_{d}$-functor such that $\widetilde{\phi}_{1}\left(V^{\otimes d}\right)=\phi_{1}$ (the existence and uniqueness of such a $\Sigma_{d}$-transformation follows from the counterpart of Lemma 3.5 for exterior powers which is obviously true). Therefore, when we put $\gamma:=\operatorname{coker}\left(\widetilde{\phi}_{1}\right)$, we get

$$
\operatorname{Ext}^{*}\left(S_{\mu}^{(i)}, S_{\lambda}^{(i)}\right)=\gamma^{\#}\left(s_{\lambda}\left(B_{i}\right)\right) .
$$

Thus we have obtained the description of the Ext's in terms similar to those used in Theorem 6.1. One should remember however, that although $\gamma$ is a quite explicitly defined symmetrization of $W_{\mu}$, one cannot expect that $\gamma \simeq w_{\mu}$ and even that $\gamma^{\#}\left(s_{\lambda}\left(B_{i}\right)\right) \simeq w_{\mu}\left(s_{\lambda}\left(B_{i}\right)\right)$, for $s_{\lambda}\left(B_{i}\right)$ is not a $Y$-permutative module.

\section{Acknowledgements}

This article (together with [6,7]) contains results of my $\mathrm{PhD}$ thesis, written under the supervision of Stanisław Betley at the University of Warsaw. I wish to express my gratitude to professor Betley for many valuable suggestions and strong encouragement. I am also grateful to the referee for drawing my attention to [20, Appendix A].

\section{REFERENCES}

[1] Akin K., Buchsbaum D., Weyman J., Schur functors and Schur complexes, Adv. Math. 44 (1982) 207-278.

[2] BetLey S., K-theory of finite fields, K-Theory 17 (2) (1999) 103-111.

[3] Boffi G., Characteristic-free decomposition of skew Schur functors, J. Algebra 125 (1989) 288-297.

[4] Bökstedt M., The topological Hochschild homology of $\mathbf{Z}$ and $\mathbf{Z} / p$, Preprint.

[5] Breen L., Extensions du groupe additif sur le site parfait, Publ. Inst. Hautes Études Sci. 48 (1978) 39-125.

[6] ChaŁuPNIK M., Schur-De-Rham complex and its cohomology, J. Algebra 282 (2004) 699-727.

[7] ChaŁupnik M., Extensions of Weyl and Schur functors, Preprint, http://hopf.purdue.edu/cgibin/generate?/Chalupnik/extws.

[8] CHAŁUPNiK M., Koszul duality and extensions of exponential functors, Preprint, http://hopf.purdue.edu/cgi-bin/generate?/Chalupnik/koeks.

[9] Cline E., PARshall B., Scott L., Finite dimensional algebras and highest weight categories, J. reine angew. Math. 391 (1988) 85-99.

[10] Franjou V., Extensions entre puissances extérieures et entre puissances symétriques, J. Algebra 179 (1996) 501-522.

[11] Franjou V., Friedlander E., Scorichenko A., Suslin A., General linear and functor cohomology over finite fields, Ann. of Math. 150 (2) (1999) 663-728.

[12] Franjou V., Lannes J., Schwartz L., Autour de la cohomologie de MacLane des corps finis, Invent. Math. 115 (1994) 513-538.

[13] Friedlander E., Suslin A., Cohomology of finite group schemes over a field, Invent. Math. 127 (1997) 209-270.

[14] Henn H.W., LANnes J., Schwartz L., The categories of unstable modules and unstable algebras over the Steenrod algebra modulo nilpotent objects, Amer. J. Math. 115 (5) (1995) 1053-1106.

[15] James G., Kerber A., The Representation Theory of the Symmetric Group, Encyclopedia of Mathematics and Its Applications, Addison-Wesley, Reading, MA, 1981.

[16] KuHn N., Generic representations of the finite general linear groups and the Steenrod algebra I, Amer. J. Math. 116 (1994) 327-360. 
[17] KUHN N., Generic representations of the finite general linear groups and the Steenrod algebra II, K-Theory 8 (4) (1994) 395-428.

[18] KuHn N., Generic representations of the finite general linear groups and the Steenrod algebra III, K-Theory 9 (3) (1995) 273-303.

[19] MarTin S., Schur Algebras and Representation Theory, Cambridge University Press, Cambridge, 1993.

[20] Macdonald I., Symmetric Functions and Hall Polynomials, Clarendon Press, Oxford, 1979.

[21] Piriou L., Schwartz L., Extensions de foncteurs simples, K-Theory 15 (1998) 269-291.

[22] Troesch A., Une résolution injective des puissances symétriques tordues, Annales de l'Institut Fourier, submitted for publication.

(Manuscrit reçu le 23 juillet 2004; accepté, après révision, le 15 juillet 2005.)

\footnotetext{
Marcin CHAŁUPNIK

Instytut Matematyki,

University of Warsaw,

ul. Banacha 2, 02-097 Warsaw,

$$
\text { Poland }
$$

E-mail:mchal@mimuw.edu.pl
} 